\title{
Expression of lysyl oxidase in human osteosarcoma and its clinical significance: A tumor suppressive role of LOX in human osteosarcoma cells
}

\author{
XIN XU, BIN WANG and YANLING XU \\ Department of Orthopedic Surgery, Shanghai No. 6 People's Hospital, Shanghai Jiaotong University \\ School of Medicine, Shanghai 200233, P.R. China
}

Received June 7, 2013; Accepted July 18, 2013

DOI: $10.3892 /$ ijo.2013.2067

\begin{abstract}
Lysyl oxidase (LOX) is an extracellular matrix (ECM) remodeling enzyme, which is involved in the development and progression of many types of tumors. LOX dysfunction is observed in colorectal, breast and ovarian cancer. However, the precise effects and molecular mechanisms of LOX action in osteosarcoma progression are still unknown. We evaluated the role of LOX in human osteosarcoma cell lines and clinical tumor samples in order to determine the function of this molecule. In our study, we showed that the expression level of LOX mRNA and protein were decreased in human osteosarcoma tissues as compared with normal tissue samples. In addition, we employed adenovirus-mediated overexpression of LOX in U-2OS and HOS cells to investigate the role of LOX in osteosarcoma cell lines. Adenovirus-mediated overexpression of LOX could efficiently increase the expression levels of LOX in osteosarcoma cell lines at both mRNA and protein levels. Increased expression of LOX inhibited the proliferation and migration of human osteosarcoma cells and promoted its apoptosis. Moreover, the Ki-67 and PCNA expression was decreased and MMP-2 and MMP-9 expression was inhibited. These findings also indicated that the effects of LOX may be mediated via the PI3K/AKT signaling pathway since LOX-mediated functions could be blocked by $\beta$-aminopropionitrile ( $\beta$-APN), a LOX inhibitor. Taken together, our data indicated that LOX may be a tumor suppressor and could be regarded as a therapeutic target in human osteosarcoma.
\end{abstract}

\section{Introduction}

Osteosarcoma is the most common, frequent and refractory malignant bone tumor (1). Although various treatment

Correspondence to: Professor Yanling Xu, Nursing Administrative Department, Shanghai No. 6 People's Hospital, Shanghai Jiaotong University School of Medicine, 600 Yishan Road, Shanghai 200233, P.R. China

E-mail: xuyanling317@163.com

Key words: lysyl oxidase, osteosarcoma, growth, migration, PI3K options are available, it is still a tumor with a high mortality rate ascribed to the development of metastasis to the lungs and frequent recurrence after surgery $(2,3)$. Therefore, identification of factors which are crucial for osteosarcoma progression is necessary for the development of new therapeutic strategies for the treatment of osteosarcoma.

Lysyl oxidase (LOX) is a key enzyme which is required for the normal biosynthesis of collagen and elastin maturation in the extracellular compartment $(4,5)$. It is synthesized and secreted as a $50-\mathrm{kDa}$ inactive pro-enzyme (LOX) and then is processed to a functional 32-kDa LOX enzyme and an 18-kDa LOX pro-peptide (LOX-PP). LOX pro-peptide was widely confirmed as a tumor suppressive factor (6-8) but a paradoxical role for LOX in tumorigenesis and metastasis were reported. Several studies have suggested that LOX contributes to distant metastasis (9-11) and an increased expression of LOX is positively correlated with disease progression and survival in colorectal cancer (11), prostate cancer (12), ovarian cancer (13) and renal clear cells (14). However, earlier research found that the injection of $\mathrm{LOX}^{-/}$normal rat kidney fibroblasts into nude mice could promote tumor formation and metastases to the peritoneum and lungs (15). In addition, the tumor-suppressor activity of LOX was confirmed in basal and squamous skin cell carcinoma (16). Taken together, these above studies demonstrate that LOX has both tumor suppressors and metastasis promoters and the biological response of cancer cells to LOX may depend on the particular cell type and other factors that are not yet defined.

To date, however, the exact role of LOX in osteosarcoma is not well understood (17). In the present study, we examined the endogenous expression level of LOX in human osteosarcoma cell lines and clinical tumor samples. Then, by overexpressing LOX in U-2OS and HOS cells we analyzed the effects of LOX on osteosarcoma cell proliferation, cell cycle, apoptosis and invasion, and also explored the underlying signaling pathway.

\section{Materials and methods}

Materials. Human bone tumor samples $(n=95)$ were obtained as anonymous specimens and the normal bone tissues $(n=20)$ were obtained from the resected and discarded bone samples from individuals who underwent total knee arthroplasty with no history of tumors from Hospital. The study was reviewed 
and approved by the Institutional Review Board of the Hospital. $\mathrm{SaO} 2, \mathrm{U}-2 \mathrm{OS}$ and HOS human osteosarcoma cell lines used in the experiments were purchased from American Type Culture Collection (ATCC, Manassas, VA, USA). Adenovirus vector with LOX expression (adLOX), negative control vector with GFP expression (adGFP) and virion-packaging elements were purchased from Genechem Company (Shanghai, China). All primary antibodies were purchased from Cell Signaling Technologies (Beverly, MA, USA).

Reagents. $\beta$-APN was purchased from Sigma-Aldrich Chemical Co. (St. Louis, MO, USA); apoptosis detection kit (Hoechst 33258), 3-(4,5-dimethylthiazol-2-yl)-3,5-diphenyl tetrazolium bromide (MTT) and ECL-PLUS kit were purchased from Beyotime (Haimen, China); Dulbecco's modified Eagle's medium and fetal bovine serum (FBS) were purchased from Gibco (Gibco, Montevideo, Uruguay); M-MLV Reverse Transcriptase was purchased from Promega (Madison, WI, USA); TRIzol Reagent and Lipofectamine 2000 were purchased from Invitrogen (Carlsbad, CA, USA); SYBR-Green Master Mixture and the primers were purchased from Takara (Otsu, Japan).

Cell culture, adenovirus transfection and study protocol. $\mathrm{SaO} 2$, U-2OS and HOS human osteosarcoma cell lines were cultured in DMEM medium supplemented with $10 \% \mathrm{FBS}$, and antibiotics (100 IU/ml of penicillin $\mathrm{G}$ and $100 \mathrm{Ag} / \mathrm{ml}$ of streptomycin). They were all incubated at $37^{\circ} \mathrm{C}$ humidified atmosphere of $5 \% \mathrm{CO}_{2}$ and the medium was replaced every 2 days. U-2OS and HOS cell lines were infected with recombinant adenovirus vector expressing LOX or GFP (adLOX and adGFP). In preliminary experiments, cells were co-infected with recombinant adenovirus vector adLOX or negative control adGFP at MOIs of 10-100 pfu/cell according to the manufacturer's instructions. Cells were subcultured at a 1:5 dilution in $400 \mu \mathrm{g} / \mathrm{ml} \mathrm{G} 418$-containing medium and positive stable transfectants were selected. In this study, a recombinant adenovirus vector containing a LOX gene and the negative control adenovirus vector were used to transfect osteosarcoma cells. U-2OS and HOS cells without gene transfection were used as a control group. Subsequently, followed by 24-h recovery after transfection, the proliferation, metastasis and invasion were assessed, respectively.

Real-time PCR. To quantitatively determine the mRNA expression level of LOX in vivo and in vitro, real-time PCR using SYBR-Premix Ex Taq (Takara, Shiga, Japan) was used according to the manufacturer's protocol. Total RNA of each specimen was extracted with TRIzol and assessed by an ABI Prism 7500 sequence detection system (Applied Biosystems, San Francisco, CA, USA). The genes were amplified using specific oligonucleotide primer and human $\beta$-actin (actin) gene was used as an internal control. The PCR primer sequences were as follows: LOX, 5'-ACTGCACACACACAGGGATTG-3' and 5'-GCCTT CTAATACGGTGAAATTG-3'; $\beta$-actin, 5 '-CTGGGACGACA TGGAGAAA-3' and 5'-AAGGAAGGCTGGAAGAGTGC-3'; Data were analyzed using the comparative $\mathrm{Ct}$ method $\left(2^{-\Delta \Delta \mathrm{Ct}}\right)$.

Immunohistochemistry. The localization of LOX protein in bone tumor tissues and normal tissues were assessed by immunohistochemistry. Bone tumor tissues and normal tissues excised from patients were embedded in paraffin and cut into $6 \mu \mathrm{M}$ sections. After deparaffinization, the sections were incubated in $3 \% \mathrm{H}_{2} \mathrm{O}_{2}$ and $0.1 \%$ Triton $\mathrm{X}-100$ and microwaved in Tris-buffer for $10 \mathrm{~min}$ to retrieve the antigen. The sections were then blocked in 5\% goat serum (Invitrogen) for $30 \mathrm{~min}$ and incubated with LOX antibody (diluted 1:200) for $2 \mathrm{~h}$ at room temperature. While a negative control was performed using a section without primary antibody. Following incubation with secondary antibodies, bound antibodies were visualized by a Power Vision Two-step Histostaining Reagent (Dako, Carpinteria, CA, USA) and antibody binding was visualized by incubation with DAB (Boster, Wuhan, China) for $3 \mathrm{~min}$ at room temperature followed by counterstaining with hematoxylin (Basa, Zhuhai, China). The LOX immunoreactive and non-immunoreactive cells with a nucleus were counted in 3 sections (with interval 3 sections) per patient in each group. The positive staining of LOX was shown as brown particles. The sections were observed and photographed with the optical microscope (Olympus, Tokyo, Japan). A percentage was calculated by the immunostained/total neuronal ratio x $100 \%$.

Western blot assay. The cells were harvested and extracted at indicated time using lysis buffer (Tris- $\mathrm{HCl}$, mercaptoethanol, SDS and glycerol). Cell extracts were boiled for $8 \mathrm{~min}$ in loading buffer and then equal amount of cell extracts were separated on $8-15 \%$ SDS-PAGE gels according to the molecular weight. Separated protein bands were transferred into polyvinylidene fluoride (PVDF) membranes and the membranes were blocked in 5\% skim milk powder. The primary antibodies against LOX, Ki-67, PCNA, COX-2, MMP-2, MMP-9, PI3Kp85 $\alpha$ and p-AKT were diluted according to the instructions of antibodies and incubated overnight at $4^{\circ} \mathrm{C}$. Then, horseradish peroxidase-linked secondary antibodies were added at a dilution ratio of 1:800, and incubated at room temperature for $3 \mathrm{~h}$. The membranes were washed with PBS for three times and the immunoreactive bands were visualized using ECL-PLUS kit according to the kit instructions. The relative protein level in different cell lines was normalized to $\beta$-actin (actin) concentration.

Cell proliferation assay. Cell proliferation was analyzed with the MTT assay. Briefly, the cells were incubated in 96-well plates at a density of $1 \times 10^{5}$ cells per well with DMEM medium supplemented with $10 \%$ FBS. Cells were treated with $20 \mu \mathrm{l}$ MTT dye at $0,12,24,48,72$ and $96 \mathrm{~h}$ and then incubated with $150 \mu \mathrm{l}$ of DMSO for $5 \mathrm{~min}$ ). The absorbance of optical densities at each time point was measured at $570 \mathrm{~nm}$ with enzyme immunoassay analyzer (Bio-Rad, Hercules, CA, USA).

Cell cycle analysis. For cell cycle analysis, $10^{6}$ cells were harvested, re-suspended, washed with phosphate-buffered saline and fixed in $70 \%$ ethanol. Cells were then treated with RNase $(10 \mu \mathrm{g} / \mathrm{ml})$ and propidium iodide $(50 \mu \mathrm{g} / \mathrm{ml})$ for $30 \mathrm{~min}$. The cell cycle phase distribution was determined by analytical DNA flow cytometry. The percentage of cells in each phase of the cell cycle was analyzed using Modfit software (Verity Software House, Topsham, ME, USA).

Wound-healing assay. U-2OS and HOS cells were plated in each well of a 6-well culture plate and allowed to grow to $80 \%$ confluence. Treatment with adLOX was then performed. On 
A
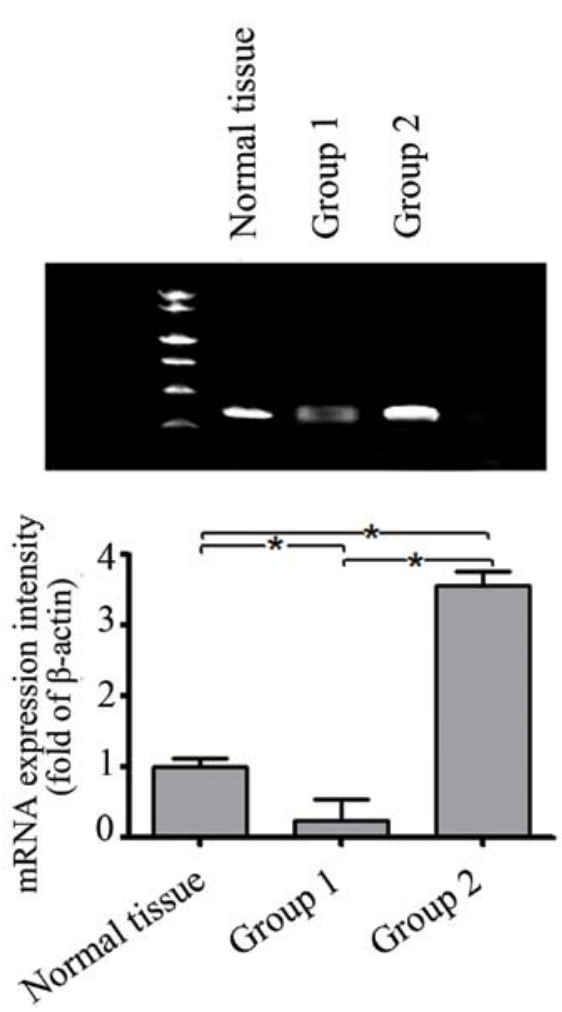

B
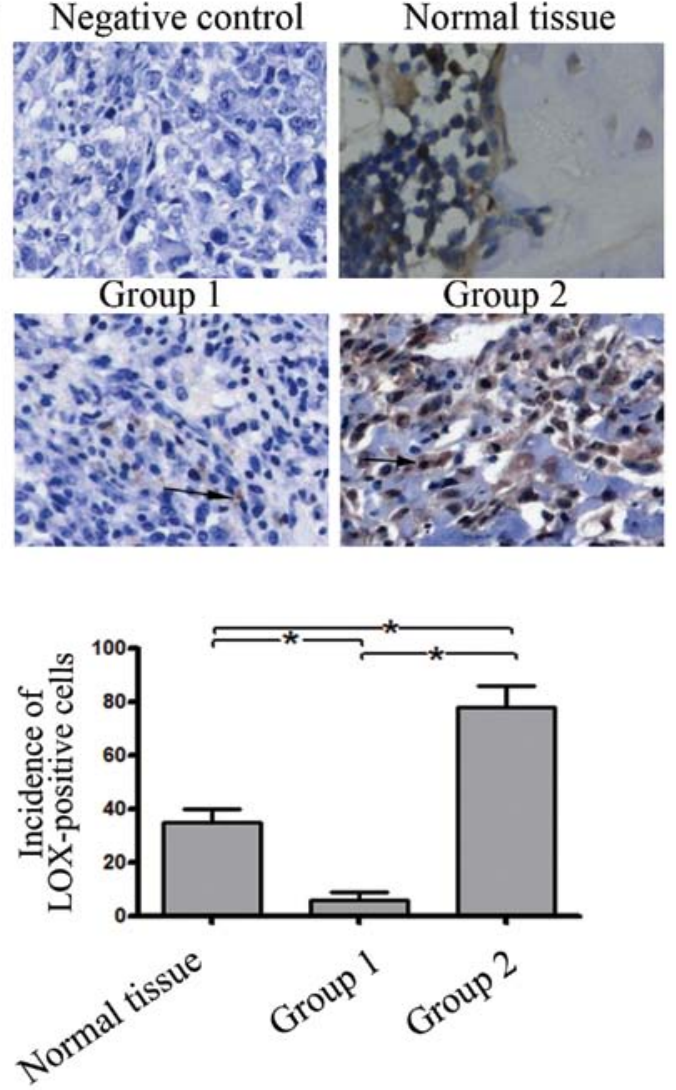

Figure 1. LOX abnormal expression is detected in human bone tumor tissues. (A) LOX mRNA expression in human bone tumor and normal bone tissues were detected using RT-PCR. The representative data derived from paired tumor and normal tissue samples presented here high level and low level of LOX mRNA expression. (B) Immunohistochemical localization of LOX in human bone tumor and normal tissues. The tumor tissue pairs investigated in (B) were divided into two subgroups according to RT-PCR results. A relative low expression level of LOX in group 1 and a relative high expression level in group 2 were observed as compared with normal tissue group. For negative control, corresponding sections of each tissue block were processed in the same way, except the primary antibody ${ }^{*} \mathrm{P}<0.05$ ).

the next day, a wound was created using a $10 \mu$ l micropipette tip. The migration of cells towards the wound was monitored at 24 and $48 \mathrm{~h}$.

Transwell invasion assay. Briefly, the cells were removed by trypsinizing and suspended with medium supplemented with $10 \%$ FBS, then $1 \times 10^{5}$ were added into the Transwell innserts with $8-\mu \mathrm{m}$ pore size (BD Biosciences) for 24 -well plates coated with $50 \mu \mathrm{l}$ Matrigel (BD Biosciences), and $200 \mu \mathrm{l}$ medium containing $15 \%$ FBS were added in the bottom chamber. Undergoing migration for 24 and $48 \mathrm{~h}$, a cotton swab was used to remove the non-migrated cells in the upper chamber then the filters were individually fixed with $4 \%$ paraformaldehyde and were determined with hematoxylin and eosin staining. The cell number was counted in five random fields of each chamber under the microscope.

Hoechst 33342 assay. The cells were prepared at a density of 5,000 cells per well in a 24-well plate. Apoptotic cells were detected by using the Hoechst 33258 staining. The cells were fixed with $4 \%$ paraformaldehyde for $10 \mathrm{~min}$, washed with PBS for three times and then stained with $2 \mu \mathrm{g} / \mathrm{ml}$ Hoechst 33258 for $5 \mathrm{~min}$. Morphologic changes in apoptotic nuclei were evaluated under a fluorescence microscope (excitation wavelength $350 \mathrm{~nm}$, emission filter $460 \mathrm{~nm}$ ) (FluoView, Olympus).
Statistical analysis. All data are presented as the means \pm standard error (SE) for at least three independent experiments. T-test was used for two group comparison and one-way analysis of variance (ANOVA) using Fisher's exact test was employed for multiple comparisons. The LSD method of multiple comparisons was used when the probability for ANOVA was statistically significant. $\mathrm{P}<0.05$ was considered with statistical significance.

\section{Results}

Low mRNA and protein expression of LOX in advanced-stage human osteosarcoma tissues. To ascertain whether abnormal LOX expression occurs in osteosarcoma tissues, the mRNA levels of LOX were examined in tumor/normal paired tissue samples by RT-PCR analysis (95/20). As a result, the expression level of LOX in $18 \%$ (17/95) was similar to that of the mean level of normal tissues ( $>0.5$-fold and $<2$-fold). However, the expression level of LOX in 57\% (55/95) of the tumor tissue was significantly lower than that of the mean level of normal tissues $(<0.5$-fold). A higher level of LOX expression ( $>2$-fold) was observed in 30\% (23/78) of tumor tissues (Fig. 1A). On the basis of the results of real-time PCR, the cases were divided into group 1 (55 cases, low level of LOX expression) and group 2 (23 cases, high level of LOX). 
A

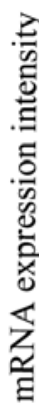

C

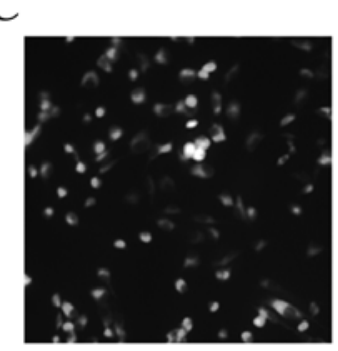

U-2OS

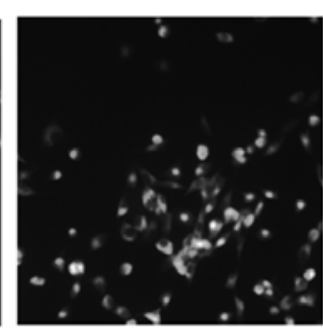

HOS

E

B
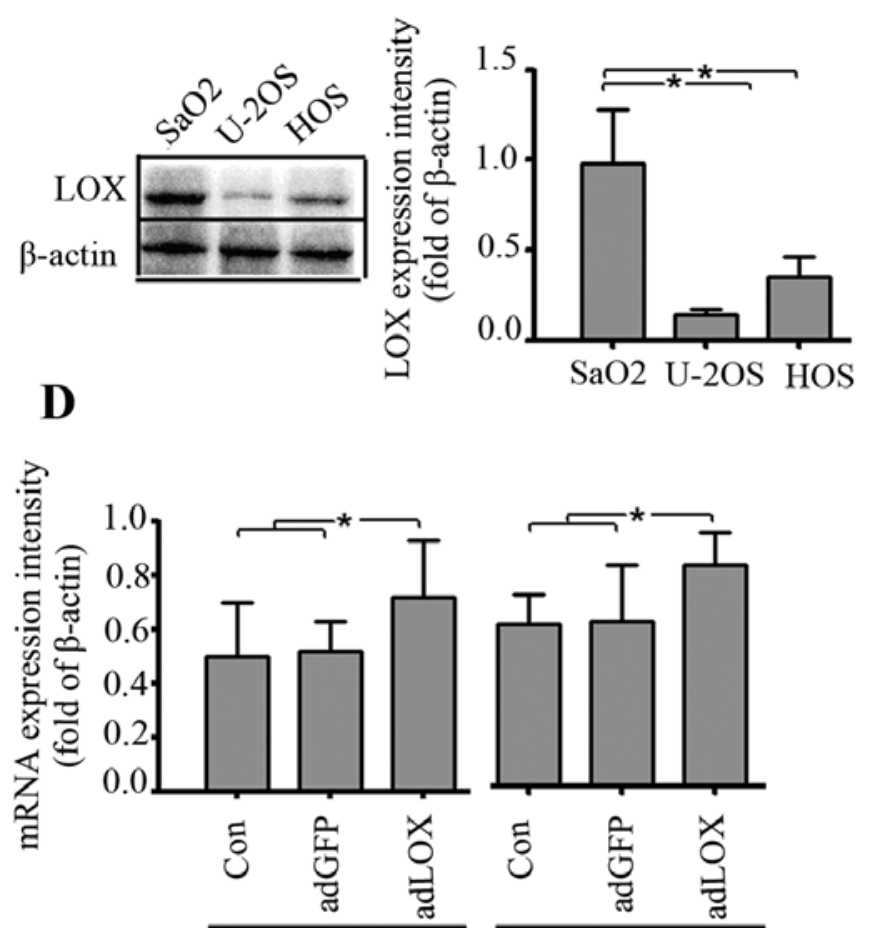

$\mathrm{U}-2 \mathrm{OS}$

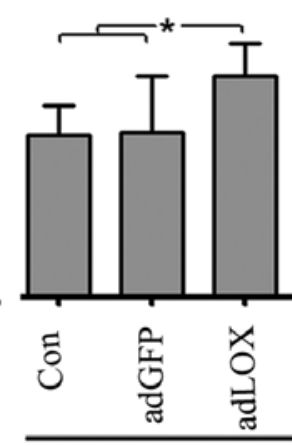

HOS
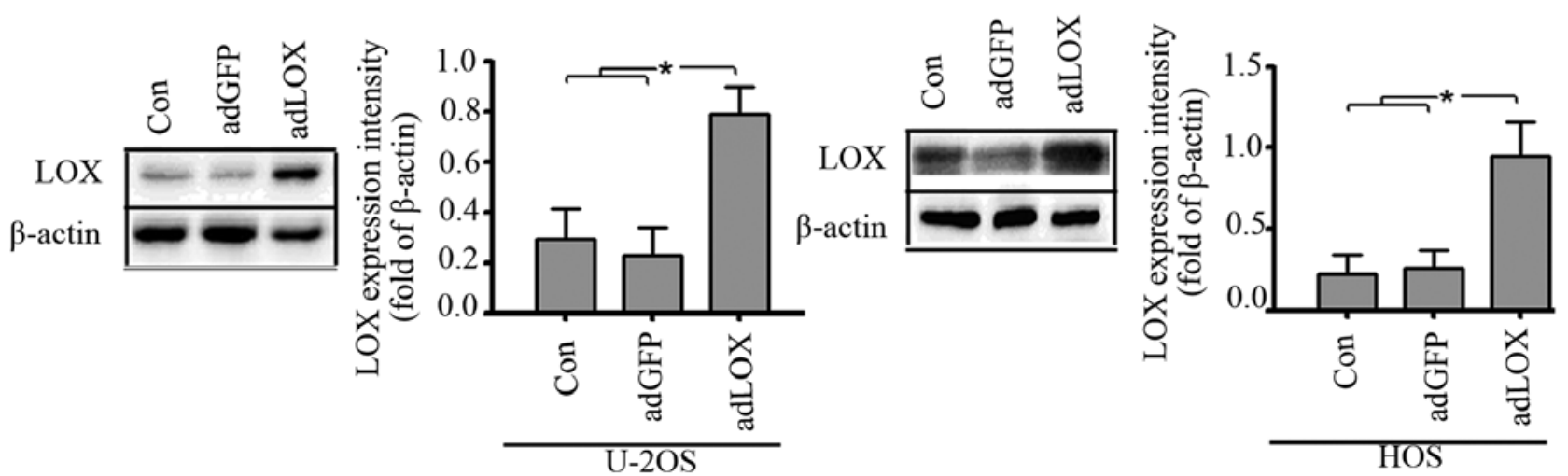

Figure 2. The expression of LOX in adLOX U-2OS and HOS cell lines. (A) Real-time-PCR and (B) western blot analysis results showed the endogenous expression of LOX mRNA and protein in SaO2, U-2OS and HOS cell lines. (C) Adenovirus vector adLOX constructed was used for infecting the U-2OS, and HOS cell lines. (D) Real-time PCR and (E) western blot analysis showed that a significant increase of LOX expression was observed in adLOX group compared with the adGFP group and Con group $\left({ }^{*} \mathrm{P}<0.05\right)$.

In addition, the level and localization of LOX protein were further assessed by immunohistochemisty in groups 1 and 2. Consistently, the incidence of LOX-positive cells in the tumor tissues and the mean level of LOX protein expression in group 1 were significantly lower than those in the normal tissues (Fig. 1B). In contrast, the incidence of LOX-positive cells and the mean level of LOX protein expression in group 2 were higher than those in the normal tissues (Fig. 1).

Subsequently, to further evaluate whether the expression levels of LOX mRNA and protein were related to clinical therapeutic outcomes, the clinical status of each specimen was determined according to tumor-node-metastasis classification (TNM) (Table I). Our results indicated that the tumor size in group 1 was significantly larger than that in group 2 and there were more cases in the poor nodal status in group 2 . In addition, the patients in group 2 had a significantly higher occurrence of pulmonary metastasis. These results revealed a decrease in LOX mRNA expression in most advanced-stage tumor tissues.

The expression of LOX in osteosarcoma cell lines and construction of LOX overexpression vector. The endogenous expression of LOX in human osteosarcoma cell lines, $\mathrm{SaO} 2$, U-2OS and HOS, was first evaluated using RT-PCR and western blot analysis. As shown in Fig. 2A and B, there were different levels of mRNA and protein expression of LOX in $\mathrm{SaO} 2, \mathrm{U}-2 \mathrm{OS}$ and HOS cell lines, but the expression levels of LOX were significantly higher in $\mathrm{SaO} 2$ cell line than those in U-2OS and HOS cell lines. Thus, U-2OS and HOS were used as osteosarcoma cell lines for infection by recombinant adenovirus containing LOX gene (Fig. 2A and B). 
Table I. The analysis of prognostic factors according to LOX mRNA expression.

\begin{tabular}{lccc}
\hline & $\begin{array}{c}\text { Group 1 } \\
\text { (no. of cases) }\end{array}$ & $\begin{array}{c}\text { Group 2 } \\
\text { (no. of cases) }\end{array}$ & P-value \\
\hline Age (years) & $18.9 \pm 5$ & $22.8 \pm 6.7$ & 0.19 \\
Gender & & & \\
Male & 38 & 19 & 0.27 \\
Female & 17 & 4 & \\
Size of tumor & & & \\
T1 & 17 & 12 & 0.23 \\
T2 & 25 & 6 & \\
T3 & 16 & 5 & \\
T4 & 1 & 0 & \\
Nodal status & & & \\
N0 & 43 & 23 & 0.04 \\
N1 & 10 & 0 & \\
N2 & 2 & 0 & \\
N3 & 0 & 0 & \\
Metastasis & & & \\
M0 & 39 & 23 & 0.16 \\
M1 & 16 & 0 & \\
5-year survival & & & \\
Alive & 21 & 16 & \\
Dead & 34 & 7 & \\
\hline
\end{tabular}

In order to enhance the exogenous expression of LOX in osteosarcoma U-2OS and HOS cells, recombinant adenovirus containing LOX gene (adLOX) was constructed and used for upregulation of LOX in the U-2OS and HOS cell lines. The infection efficiency of adLOX (at a multiplicity of infection $=100$ ) in U-2OS and HOS cell lines were both greater than $80 \%$ by fluorescence microscopy (Fig. 2C). Real-time PCR and western blot assays were performed to measure the exogenous expression of LOX after $48 \mathrm{~h}$ following adenovirus infection and an obvious increase of LOX mRNA and protein expression was observed in adLOX group as shown in Fig. 2D and E.

Proliferation of osteosarcoma cells was inhibited by upregulation of LOX expression. Deregulation of cell proliferation and cell cycle is one of the hallmarks of cancer (18). In order to detect the effect of LOX on cell proliferation, we investigated the proliferative activities of adLOX-infected U-2OS and HOS cells by MTT assay. As a result, the cell proliferative ability of adLOX-infected U-2OS and HOS was significantly suppressed as compared with the adGFP group and control group. It was indicated that LOX overexpression would reduce the proliferative activities of U-2OS and HOS cell lines. However, no difference was found between adGFP group and control group in U-2OS and HOS cell lines (Fig. 3A). To further confirm whether LOX overexpression inhibited cell cycle progression, flow cytometry was employed. As shown in Fig. 3B, the apoptotic incidence of U-2OS and HOS cells in the adLOX group was remarkably higher than that in the adGFP group and control
A
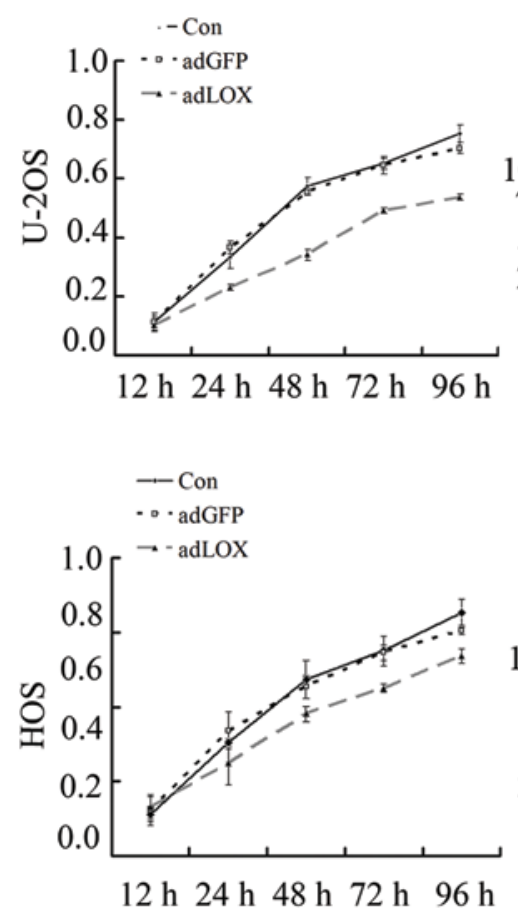

B
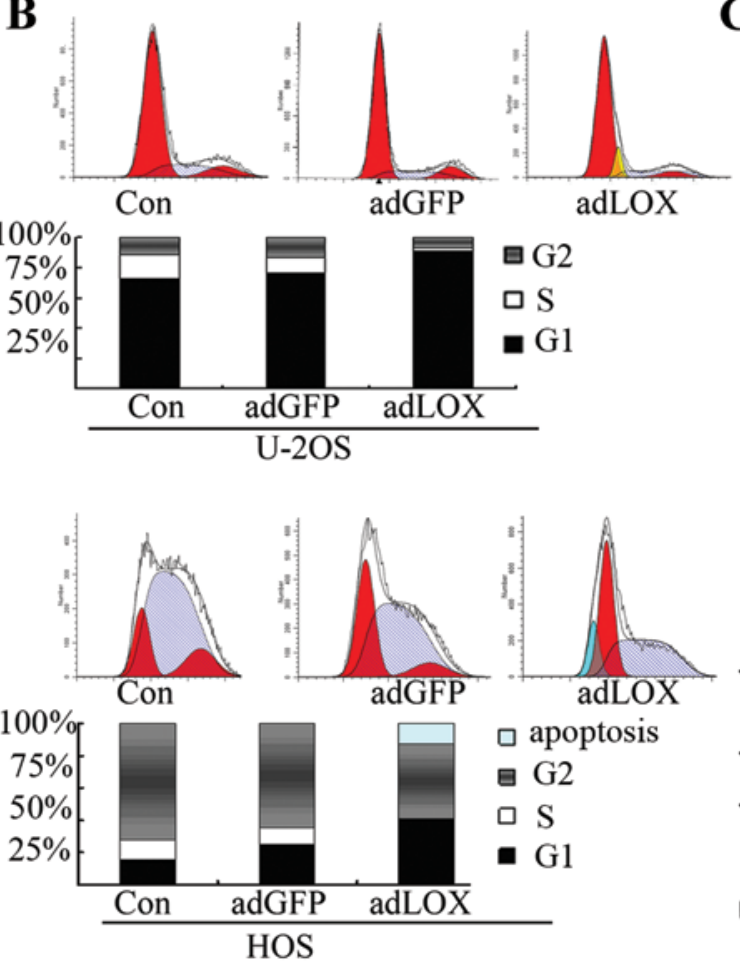

C Con adGFP adLOX

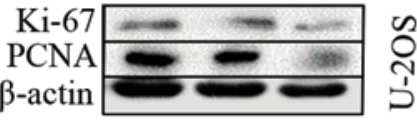

$\beta$-actin
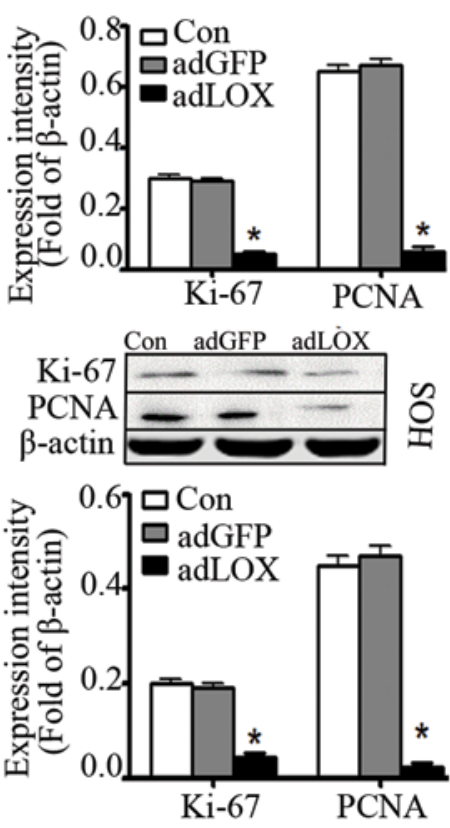

Figure 3. Effect of adLOX on proliferation and cell cycle distribution in U-2OS and HOS cell lines. (A) It was indicated by MTT assay that adLOX significantly reduced the proliferative activities of U-2OS and HOS cell lines in a time-dependent manner compared with the adGFP group and CON group. (B) FACS analysis demonstrated that overexpression of LOX causes G0/G1 arrest and apoptosis in U-2OS and HOS cell lines. (C) The expression of Ki-67 and PCNA was examined by western blot assay, indicating that the amount of Ki-67 and PCNA expression was significantly decreased in adLOX group compared with the adGFP group and Con group in $\mathrm{U}-2 \mathrm{OS}$ and HOS cell lines $(\mathrm{P}<0.05)$. 
A
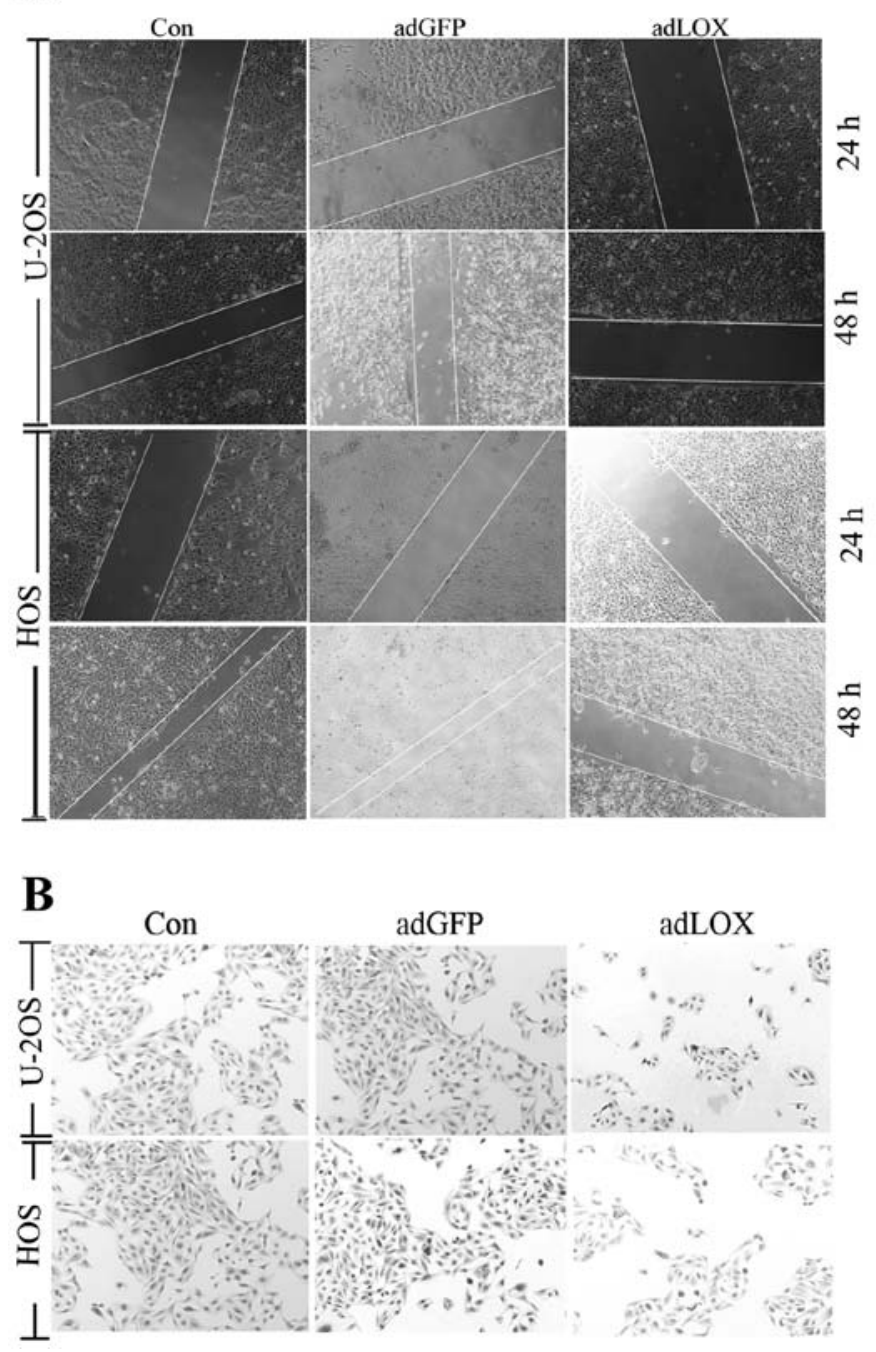

C
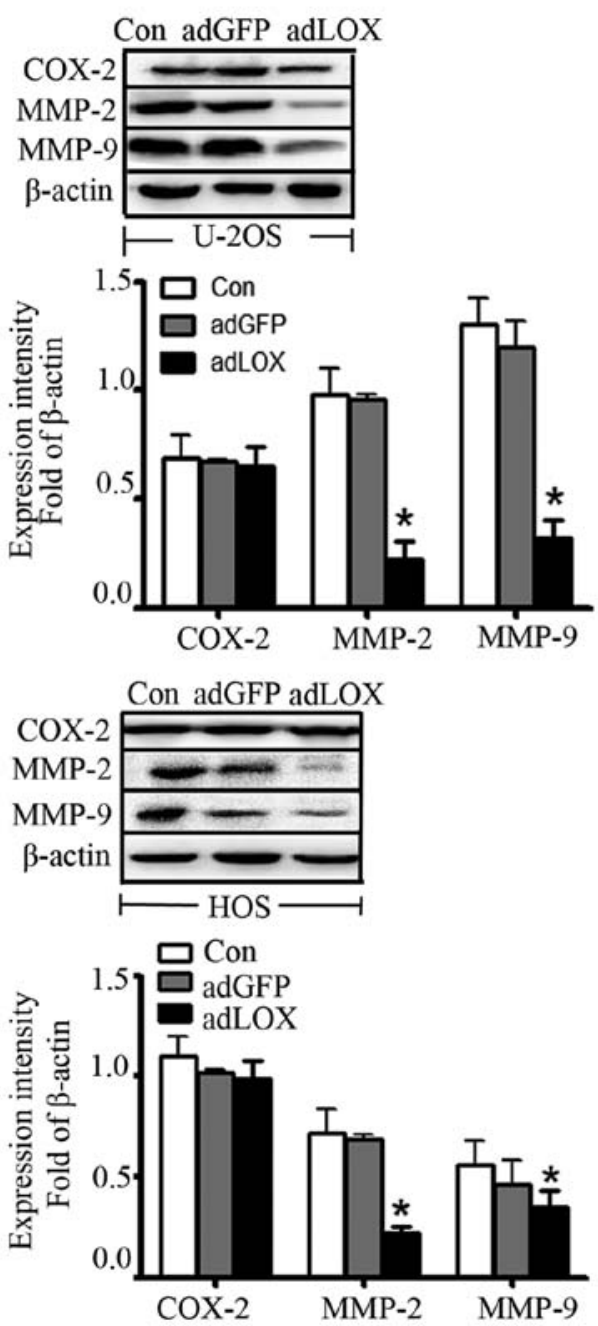

Figure 4. Inhibition of osteosarcoma cell migration and invasiveness by adLOX in U-2OS and HOS cell lines. (A) Wound-healing assay showed that the migration capacities of osteosarcoma cells in adLOX group were markedly lower than those in adGFP group and Con group. (B) Transwell invasion assay for the transmembrane ability of each group of cells and quantitative results showed an impaired ability in adLOX U-2OS and HOS cell lines. (C) Western blot assay was performed to examine the effect of LOX overexpressing on MMP-9, MMP-2 and COX-2 expression, showing that the expression of MMP-9 and MMP-2 protein was significantly inhibited in adLOX group compared with the ad-GFP group and CON group in MG-63 and HOS cell lines ( $\mathrm{P}<0.05$ ).

group. Cell cycle kinetics showed that the G0/G1 phase fraction was increased, while $\mathrm{S}$ phase fraction was decreased. Cell cycle was arrested in G0/G1 phase more in the adLOX group than in the adGFP group and control group (Fig. 3B).

In order to investigate the mechanisms of proliferative inhibition of LOX, we evaluated effects of LOX on two independent proliferative markers: Ki-67 and proliferating cell nuclear antigen (PCNA). Ki-67 is identified as a critical mediator in tumor progression in Ewing's sarcoma (19) and is widely used to evaluate the outcome of anticancer treatment $(1,20,21)$. Similarly, PCNA has been employed to evaluate cell proliferation (22). The expression of Ki-67 and PCNA was examined by western blot assay and the results indicated that the expression level of Ki-67 and PCNA was significantly inhibited by LOX overexpression in U-2OS and HOS cell lines and no difference was found between adGFP group and control group (Fig. 3C). These data suggested that overexpression of LOX might inhibit osteosarcoma cell proliferation via downregulation of Ki-67 and PCNA.
Cell migration was suppressed by LOX. To determine the effect of LOX on U-2OS and HOS cell migration, wound-healing assay was performed and the results showed that the migrative capacities of osteosarcoma cells with adLOX infection were markedly suppressed. However, no significant changes were detected between adGFP group and control group (Fig. 4A). Moreover, Transwell invasion assay was performed to examine the ability of adLOX-infected 2U-2OS and HOS cells to traverse the Matrigel-coated Transwell chamber. As shown in Fig. 4B, compared with the adGFP group and control group, invasion through Matrigel in adLOX group was reduced by $48 \%$.

It has been reported that cyclooxygenase-2 (COX-2) (23) and MMPs were correlated with tumor invasive progression or metastasis in several types of cancers $(22,24,25)$. To investigate whether the activities of cyclooxygenase-2 and MMPs were affected by LOX, western blot analysis was used to examine the expressions of COX-2, MMP-9 and MMP-2. As shown in Fig. 4C, the expression of MMP-9 and MMP-2 proteins was 
A

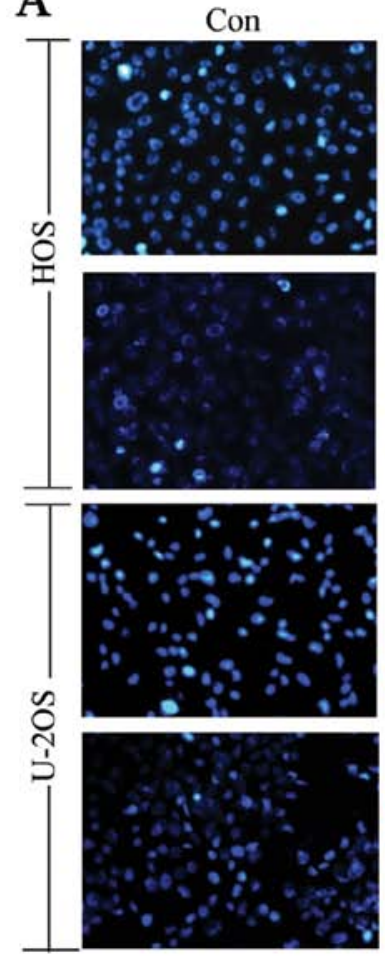

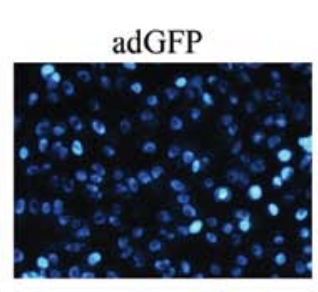
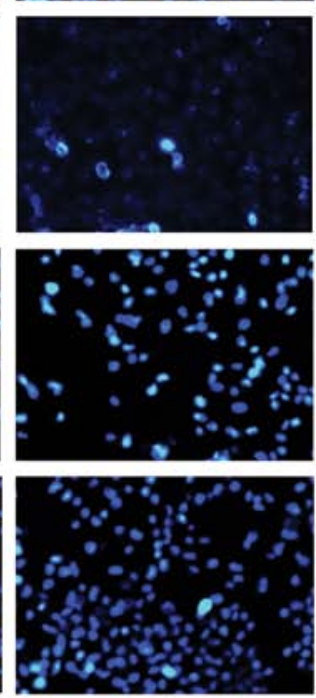
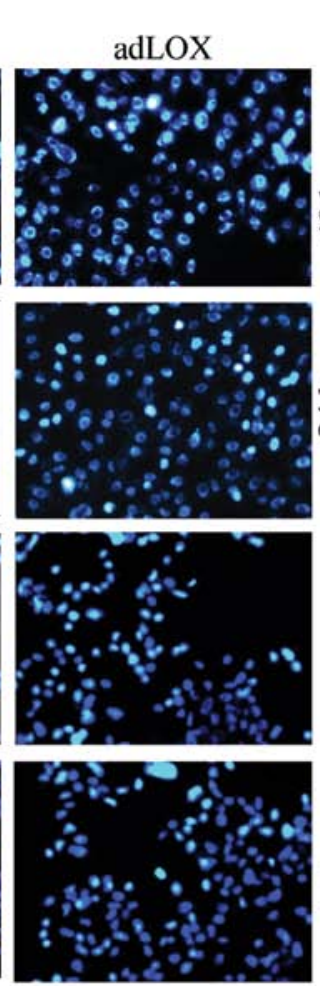

B

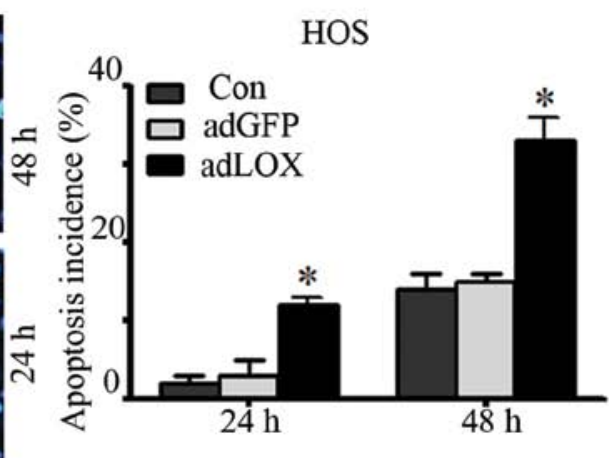

$\mathrm{U}-2 \mathrm{OS}$

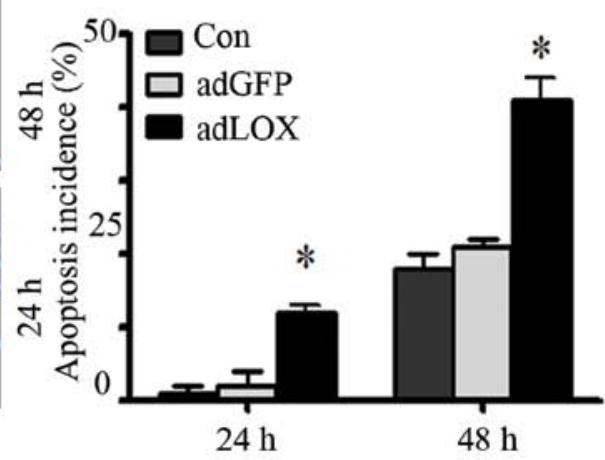

Figure 5. Induction of osteosarcoma cell apoptosis by serum deprivation. At the end of the incubation period for 24 and $48 \mathrm{~h}$, the U-2OS and HOS cells were stained with Hoechst 33342 staining and their cell nucleus was observed under the microscope for apoptosis, showing that the number of apoptotic cells (strong blue staining) in adLOX group significantly increased compared with that in the adGFP group and Con group in a time-dependent manner. Representative micrographs are showed in $(\mathrm{A})$ and quantitative scoring of all data in $(\mathrm{B})\left({ }^{*} \mathrm{P}<0.05\right)$.

significantly suppressed in adLOX group as compared with the adGFP group and control group. However, no significant difference in expression of COX-2 was found among the three groups. These data indicated that overexpression of LOX might inhibit the migration of osteosarcoma cells via downregulation of MMP-9 and MMP-2 expression.

The anti-apoptotic ability was suppressed by LOX. It has been shown that the mechanism of action of tumorigenesis factors is associated with their ability to suppress apoptosis. Thus, in this study, apoptosis was detected by Hoechst 33342 staining. After culture for 24 and $48 \mathrm{~h}$, the cells were stained with Hoechst 33342 and their cell nucleus was observed under the microscope for apoptosis. The representative micrographs (Fig. 5A) and quantitative scoring of all data (Fig. 5B) showed that the number of U-2OS and HOS apoptotic cells and necrotic cells (strong blue staining) in adLOX group significantly increased compared with that in adGFP group and control group in a time-dependent manner. No difference was found between adGFP group and control group $(\mathrm{P}>0.05)$. These data suggested that overexpression of LOX could decrease the ability of osteosarcoma cells against apoptosis.

The effect of LOX was mediated via PI3K/AKT signaling pathway. The PI3K/Akt signaling pathway plays a critical role in regulating cellular proliferation, growth, survival and motility for normal cells and its dysfunction is deeply connected with tumorigenesis $(20,26)$. To directly test the effects of PI3K/AKT signaling, the expression of PI3Kp85 $\alpha$ and p-AKT was assessed by western blot assay. As shown in Fig. 6, an obvious decrease of PI3Kp $85 \alpha$ and p-AKT was observed in adLOX group compared with that in the adGFP group and control group $\left({ }^{*} \mathrm{P}<0.05\right)$. But, no difference was found between adGFP group and control group $(\mathrm{P}>0.05)$. To further confirm this result, $300 \mu \mathrm{M} \beta$-aminopropionitrile ( $\beta$-APN), a LOX inhibitor, was used to treat the adLOX-infected U-2OS and HOS cells. Western blot results showed that the expressions of PI3Kp85 $\alpha$ and p-AKT were significantly suppressed by $\beta$-APN. In addition, the inhibitory effect of LOX on proliferation and migration of human osteosarcoma cells also could be reversed by $\beta$-APN (data not shown). These data suggested that overexpression of LOX might inhibit the proliferation and migration of human osteosarcoma cells through PI3K/AKT signaling pathway.

\section{Discussion}

LOX is a key enzyme that control extracellular matrix, collagen and elastin maturation, but, the role of LOX is not limited to these functions and it also plays a critical role in the development and invasion of osteosarcoma. A previous microarray analysis suggested that the gene expression of LOX was associated with osteosarcoma (27). Furthermore, a study in vitro identified that LOX expression could be increased by suramin (an anticancer drug) in a dose-dependent manner, indicating that suramin might inhibit tumorigenesis by increase of 

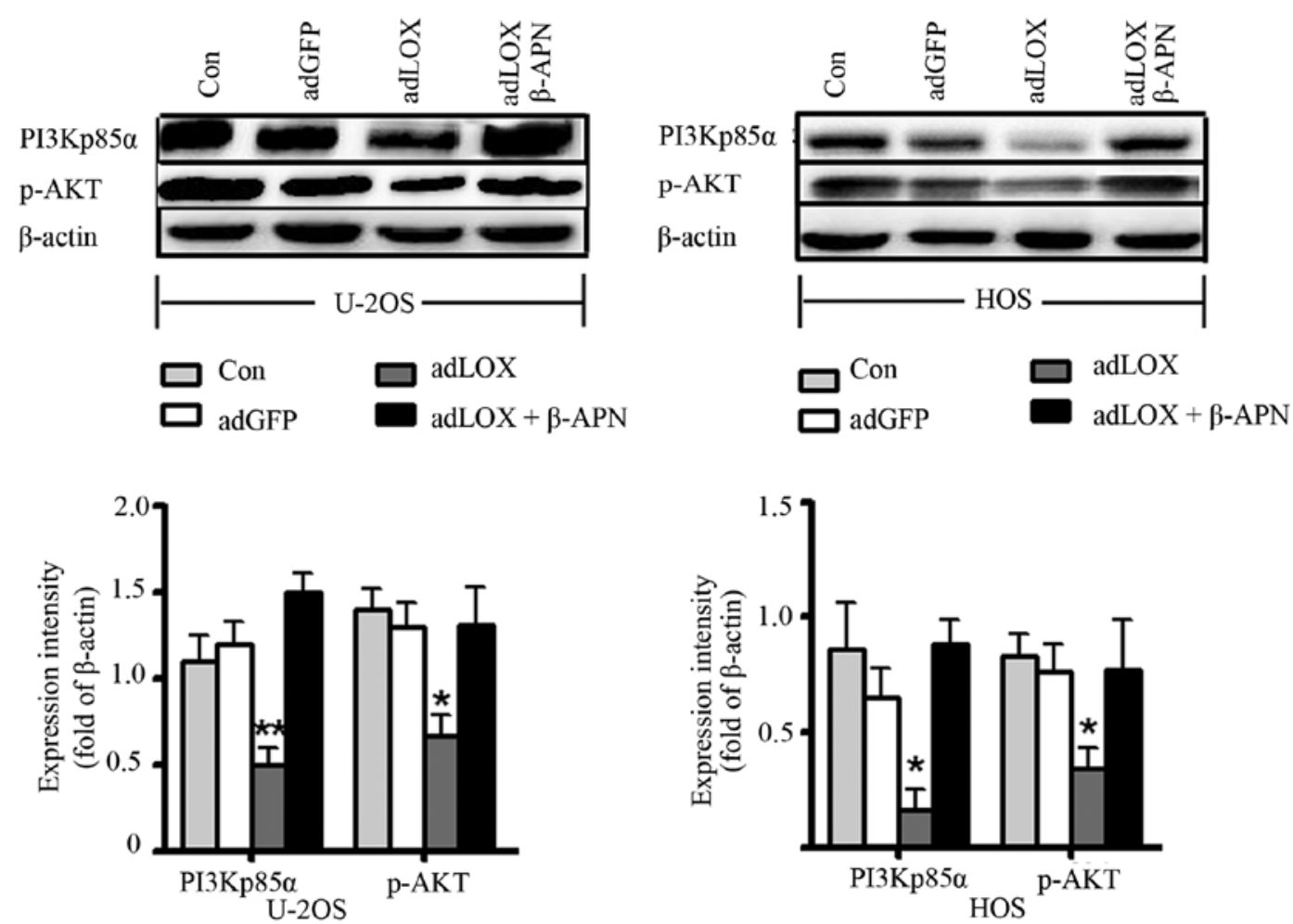

Figure 6. Western blot assays were performed at 48 -h recovery to measure the exogenous expression of PI3Kp85 $\alpha$ and p-AKT, showing an obvious decrease of PI3Kp85 $\alpha$ and p-AKT expression in adLOX group compared with the adGFP group and Con group $\left({ }^{* *} \mathrm{P}<0.01\right)$, while the effect of LOX on PI3Kp85 $\alpha$ and p-AKT was reversed by co-treatment with $\beta$-APN in adLOX group $\left({ }^{*} \mathrm{P}<0.05\right)$. No difference was found between adGFP group and Con group in U-2OS and HOS cell lines $(\mathrm{P}>0.05)$.

LOX (28). Consistently, our results also showed that patients with low LOX expression were more likely to develop poor nodal status $(\mathrm{P}=0.04)$ and survival was significantly reduced $(\mathrm{P}=0.01)$. These findings indicated that LOX might serve as a tumor suppressive gene and a prognostic marker for survival in patients with osteosarcoma.

In addition, studies have shown that LOX is upregulated in invasive breast cancer cells, and induced proliferation in vitro (29). However, our finding demonstrated that the overexpression of LOX significantly reduced the proliferative activity of U-2OS and HOS cell lines in a time-dependent manner. The result was consistent with previous reports that LOX inhibited proliferation in smooth muscle cells $(30,31)$. At the same time, we found that the expression of Ki-67 and PCNA were downregulated in U-2OS and HOS cell lines when LOX was overexpressed. These data suggested that LOX might inhibit proliferation through downregulation of Ki-67 and PCNA expression.

Failure of cells to undergo apoptosis is a feature of cancers and it has been reported that LOX has apoptosis-inducing effects in lung cancer and breast cancer cells $(6,32,33)$. Similarly, our results also identified that LOX overexpression resulted in increased apoptosis in U-2OS and HOS cell lines. On the other hand, cell cycle is a highly-ordered and tightlyregulated process involving multiple checkpoints that assesses extracellular growth signals, cell size, and DNA integrity and its deregulation would lead to tumorigenesis. Our results demonstrated that the cell cycle of osteosarcoma cells in the adLOX group had more cells arrested in G0/G1 phase and had higher apoptotic incidence compared with the adGFP group and control. Transwell invasion and wound-healing assay indicated that LOX overexpression inhibited the migration and invasiveness of osteosarcoma cells. The expression of MMP-9 and MMP-2 proteins was significantly suppressed in U-2OS and HOS cell lines with LOX overexpression. This suggested that LOX inhibited the migration of osteosarcoma cells via downregulation of MMP-9 and MMP-2 expression.

$\mathrm{PI} 3 \mathrm{~K} / \mathrm{AKT}$ is a major pathway for malignant progression in various tumors $(20,26)$. It mediated survival signals to rescue Ewing tumor cells from fibroblast growth factor 2-induced cell death (34). It was reported that several cyclooxygenase-2 inhibitors could induce tumor cell apoptosis via inhibition of PI3K/AKT signaling pathway (35,36). Furthermore, it had been reported that the LOX gene might inhibit migration and proliferation via PI3K/AKT signaling pathway in tumor cells (37-39). Our study also indicated that overexpression of LOX could lead to a marked decrease of PI3Kp85 $\alpha$ and p-AKT, accompanied by a reduced proliferative activity and migration capacity in U-2OS and HOS cell lines. The $\beta$-APN could reverse the effect of LOX on proliferation and migration of human osteosarcoma cells. This suggested that overexpression of LOX inhibited osteosarcoma cell growth and migration via blockade of the $\mathrm{PI} 3 \mathrm{~K} / \mathrm{AKT}$ signaling pathway. To our knowledge, this is the first report on the role of LOX in the growth and migration of osteosarcoma cells. But, the small sample size of 95 cases and the use of only two osteosarcoma cell lines provide limited evidence. Further research with larger sample size and more cell lines is required to confirm our findings. 
In conclusion, our investigations revealed that the expression level of LOX mRNA and protein is downregulated in human osteosarcoma tissues and the enhanced expression of LOX in osteosarcoma cells exerts inhibitory effects on growth and migration of osteosarcoma cells via blockade of the PI3K/AKT signaling pathway.

\section{References}

1. Thompson L, Wang S, Tawfik O, et al: Effect of 25-hydroxyvitamin D3 and $1 \alpha, 25$ dihydroxyvitamin D3 on differentiation and apoptosis of human osteosarcoma cell lines. J Orthop Res 30: 831-844, 2012.

2. Chugh R: Experimental therapies and clinical trials in bone sarcoma. J Natl Compr Canc Netw 8: 715-725, 2010.

3. Dai X, Ma W, He X, et al: Review of therapeutic strategies for osteosarcoma, chondrosarcoma, and Ewing's sarcoma. Med Sci Monit 17: RA177-190, 2011.

4. Kagan HM and Trackman PC: Properties and function of lysyl oxidase. Am J Respir Cell Mol Biol 5: 206-210, 1991.

5. Kim YM, Kim EC and Kim Y: The human lysyl oxidase-like 2 protein functions as an amine oxidase toward collagen and elastin. Mol Biol Rep 38: 145-149, 2011.

6. Bais MV, Nugent MA, Stephens DN, et al: Recombinant lysyl oxidase propeptide protein inhibits growth and promotes apoptosis of pre-existing murine breast cancer xenografts PLoS One 7: e31188, 2012

7. Palamakumbura AH, Vora SR, Nugent MA, et al: Lysyl oxidase propeptide inhibits prostate cancer cell growth by mechanisms that target FGF-2-cell binding and signaling. Oncogene 28 3390-3400, 2009.

8. Hurtado PA, Vora S, Sume SS, et al: Lysyl oxidase propeptide inhibits smooth muscle cell signaling and proliferation. Biochem Biophys Res Commun 366: 156-161, 2008.

9. Erler JT, Bennewith KL, Nicolau M, et al: Lysyl oxidase is essential for hypoxia-induced metastasis. Nature 440 : 1222-1226, 2006

10. Kirschmann DA, Seftor EA, Fong SF, et al: A molecular role for lysyl oxidase in breast cancer invasion. Cancer Res 62 4478-4483, 2002.

11. Baker AM, Bird D, Lang G, et al: Lysyl oxidase enzymatic function increases stiffness to drive colorectal cancer progression through FAK. Oncogene 73: 583-594, 2013.

12. Lapointe J, Li C, Higgins JP, et al: Gene expression profiling identifies clinically relevant subtypes of prostate cancer. Proc Natl Acad Sci USA 101: 811-816, 2004.

13. Wu J, Cai C, Tong D, et al: Lysyl oxidase G473A polymorphism is associated with increased risk of ovarian cancer. Genet Test Mol Biomarkers 16: 915-919, 2012.

14. Stassar MJ, Devitt G, Brosius M, et al: Identification of human renal cell carcinoma associated genes by suppression subtractive hybridization. Br J Cancer 85: 1372-1382, 2001.

15. Giampuzzi M, Botti G, Cilli M, et al: Down-regulation of lysyl oxidase-induced tumorigenic transformation in NRK-49F cells characterized by constitutive activation of ras proto-oncogene. J Biol Chem 276: 29226-29232, 2001.

16. Bouez C, Reynaud C, Noblesse E, et al: The lysyl oxidase LOX is absent in basal and squamous cell carcinomas and its knockdown induces an invading phenotype in a skin equivalent model. Clin Cancer Res 12: 1463-1469, 2006.

17. Barker HE, Cox TR and Erler JT: The rationale for targeting the LOX family in cancer. Nat Rev Cancer 12: 540-552, 2012.

18. Novello C, Pazzaglia L, Cingolani C, et al: miRNA expression profile in human osteosarcoma: role of miR-1 and miR-133b in proliferation and cell cycle control. Int J Oncol 42: 667-675, 2013.

19. Sollazzo MR, Benassi MS, Magagnoli G, et al: Increased c-myc oncogene expression in Ewing's sarcoma: correlation with Ki67 proliferation index. Tumori 85: 167-173, 1999.

20. Li B, Yang Y, Jiang S, et al: Adenovirus-mediated overexpression of BMP-9 inhibits human osteosarcoma cell growth and migration through downregulation of the PI3K/AKT pathway. Int J Oncol 41: 1809-1819, 2012.
21. Liu ZL, Wang G, Peng AF, et al: Fatty acid synthase expression in osteosarcoma and its correlation with pulmonary metastasis. Oncol Lett 4: 878-882, 2012

22. Kamei S, Sakayama K, Tamashiro S, et al: Ketoprofen in topical formulation decreases the matrix metalloproteinase-2 expression and pulmonary metastatic incidence in nude mice with osteosarcoma. J Orthop Res 27: 909-915, 2009.

23. Duan DP, Dang XQ, Wang KZ, et al: The cyclooxygenase-2 inhibitor NS-398 inhibits proliferation and induces apoptosis in human osteosarcoma cells via downregulation of the survivin pathway. Oncol Rep 28: 1693-1700, 2012.

24. Korpi JT, Hagstrom J, Lehtonen N, et al: Expression of matrix metalloproteinases-2, $-8,-13,-26$, and tissue inhibitors of metalloproteinase-1 in human osteosarcoma. Surg Oncol 20: e18-e22, 2011.

25. Zhang Y, Song L, Cai L, et al: Effects of baicalein on apoptosis, cell cycle arrest, migration and invasion of osteosarcoma cells. Food Chem Toxicol 53C: 325-333, 2012

26. Rasmussen $\mathrm{N}$ and Rathmell WK: Looking beyond inhibition of VEGF/mTOR: emerging targets for renal cell carcinoma drug development. Curr Clin Pharmacol 6: 199-206, 2011.

27. Kubista B, Klinglmueller F, Bilban M, et al: Microarray analysis identifies distinct gene expression profiles associated with histological subtype in human osteosarcoma. Int Orthop 35: 401-411, 2011.

28. Buchinger B, Spitzer S, Karlic H, et al: Lysyl oxidase (LOX) mRNA expression and genes of the differentiated osteoblastic phenotype are upregulated in human osteosarcoma cells by suramin. Cancer Lett 265: 45-54, 2008.

29. Payne SL, Fogelgren B, Hess AR, et al: Lysyl oxidase regulates breast cancer cell migration and adhesion through a hydrogen peroxide-mediated mechanism. Cancer Res 65: 11429-11436, 2005.

30. Gacheru SN, Thomas KM, Murray SA, et al: Transcriptional and post-transcriptional control of lysyl oxidase expression in vascular smooth muscle cells: effects of TGF-beta 1 and serum deprivation. J Cell Biochem 65: 395-407, 1997.

31. Peinado H, Moreno-Bueno G, Hardisson D, et al: Lysyl oxidase-like 2 as a new poor prognosis marker of squamous cell carcinomas. Cancer Res 68: 4541-4550, 2008.

32. Yu Z, Sato S, Trackman PC, et al: Blimpl activation by AP-1 in human lung cancer cells promotes a migratory phenotype and is inhibited by the lysyl oxidase propeptide. PLoS One 7: e33287, 2012

33. Palamakumbura AH, Jeay S, Guo Y, et al: The propeptide domain of lysyl oxidase induces phenotypic reversion of rastransformed cells. J Biol Chem 279: 40593-40600, 2004.

34. Hotfilder M, Sondermann P, Senss A, et al: PI3K/AKT is involved in mediating survival signals that rescue Ewing tumour cells from fibroblast growth factor 2-induced cell death. Br J Cancer 92: 705-710, 2005.

35. Liu B, Shi ZL, Feng J, et al: Celecoxib, a cyclooxygenase-2 inhibitor, induces apoptosis in human osteosarcoma cell line MG-63 via down-regulation of PI3K/Akt. Cell Biol Int 32: 494-501, 2008.

36. Jin S, Pang RP, Shen JN, et al: Grifolin induces apoptosis via inhibition of PI3K/AKT signalling pathway in human osteosarcoma cells. Apoptosis 12: 1317-1326, 2007.

37. Jeay S, Pianetti S, Kagan HM, et al: Lysyl oxidase inhibits ras-mediated transformation by preventing activation of NF-kappa B. Mol Cell Biol 23: 2251-2263, 2003.

38. Pez F, Dayan F, Durivault J, et al: The HIF-1-inducible lysyl oxidase activates HIF-1 via the Akt pathway in a positive regulation loop and synergizes with HIF-1 in promoting tumor cell growth. Cancer Res 71: 1647-1657, 2011.

39. Voloshenyuk TG, Landesman ES, Khoutorova E, et al: Induction of cardiac fibroblast lysyl oxidase by TGF-beta1 requires PI3K/Akt, Smad3, and MAPK signaling. Cytokine 55: 90-97, 2011. 